\section{Commissures, Cerebral}

Martin R. Graf

Department of Neurosurgery, Virginia

Commonwealth University Medical Center,

Richmond, VA, USA

\section{Synonyms}

Brain commissures; Commissural fibers

\section{Definition}

The right and left cerebral hemispheres are connected by three tracts of nerve fibers or axons, which are collectively referred to as commissures, cerebral. These transverse, nerve fiber bundles connect to the homologous regions of each hemisphere and are known as the corpus callosum, the anterior commissure and the posterior commissure. The corpus callosum, also referred to as the great cerebral commissure, is the largest, and it interconnects the greatest portion of the cerebral hemispheres, permitting the cerebral cortex to operate as a whole. The corpus callosum is often the site for commissurotomy, surgical bisection, to treat certain psychiatric disorders and epilepsy. The anterior commissure is a smaller bundle of nerve fibers that interconnect parts of the temporal lobes. The posterior commissure is another fiber bundle that crosses beneath the pineal gland to connect the midbrain regions of the cerebral hemispheres.

\section{Cross-References}

- Commissurotomy

- Corpus Callosum 